\title{
Acute Kidney Injury and Acute Respiratory Distress Syndrome
}

\author{
Bryan D. Park, $\mathrm{MD}^{\mathrm{a}}$, Sarah Faubel, MD ${ }^{\mathrm{b}, *}$
}

\section{KEYWORDS}

- Acute kidney injury • Acute respiratory distress syndrome • COVID-19

- Intensive care unit

\section{KEY POINTS}

- AKI is a common complication of ARDS and portends a poor prognosis.

- AKI is associated with numerous traditional and nontraditional complications that conspire to adversely affect the lungs.

- Key considerations in the management of AKI complicating ARDS include close attention to fluid balance, maintenance of euvolemia, avoidance of hypophosphatemia while on RRT, and continuous dialogue between nephrologists and critical care specialists.

- Clinicians should recognize that patients with AKI can be expected to require mechanical ventilation longer and wean longer than other patient populations.

- $\mathrm{AKI}$ is common in COVID-19 disease and is predominantly caused by sepsis pathophysiology.

\section{INTRODUCTION}

Acute kidney injury (AKI) is a common complication in patients with acute respiratory distress syndrome (ARDS) with studies reporting up to $35 \%$ incidence rate. The combination of AKI and ARDS portends worse outcomes including higher mortality and increased hospital length-of-stay. ${ }^{1-3}$ Recently, the novel SARS-CoV-2 (or COVID19) has emerged as the most significant viral pandemic in the modern era, and has further highlighted the important relationship of organ-organ crosstalk in the critically ill. In this article, we explore the interrelationship between the kidneys and the lungs in the setting of ARDS. We emphasize key clinical information including definition,

\footnotetext{
a Division of Pulmonary Sciences and Critical Care Medicine, Department of Internal Medicine, University of Colorado, Anschutz Medical Campus, 12700 East 19th Avenue, Box C272, Aurora, CO 80045, USA; ${ }^{b}$ Division of Renal Diseases and Hypertension, Department of Internal Medicine, University of Colorado, Anschutz Medical Campus, 12700 East 19th Avenue, Box C281, Aurora, CO 80045, USA

* Corresponding author.

E-mail address: sarah.faubel@cuanschutz.edu
} 
epidemiology, pathophysiology, and treatment strategies important for any critical care clinician. Finally, we also describe the current understanding of AKI in SARSCoV-2 infection given the high incidence of $\mathrm{AKI}$ in this population.

\section{DEFINITIONS OF ACUTE KIDNEY INJURY}

Early studies of hospital and intensive care unit (ICU)-acquired AKI were limited by the lack of a uniform, standard definition. ${ }^{4,5}$ Before 2004, more than 30 different definitions of AKI had been described, which created difficulties in validating diagnostic and therapeutic interventions. ${ }^{5}$ The first collaborative efforts to define and stage AKI was performed by an international, multidisciplinary group in 2004 by the Acute Dialysis Quality Initiative (ADQI) ${ }^{5}$ and then in 2005 by the Acute Kidney Injury Network. ${ }^{4}$ More recently, the Kidney Disease: Improving Global Outcomes (KDIGO) society developed rigorous evidence-based clinical practice guidelines in 2012 for the evaluation and management of $A K I .{ }^{6}$ Their proposal included a modified definition of AKI by combining the ADQI and Acute Kidney Injury Network definitions, and is now the most used definition and classification system (Table 1).

Although the 2012 KDIGO criteria for AKI have now been successfully implemented, some limitations exist. ${ }^{7}$ First, these criteria do not include identification of an underlying cause. AKI is a heterogeneous disease with a variety of causes requiring different diagnostic and therapeutic interventions. As such, the clinical context is always key, and outcomes may differ depending on the underlying cause. Second, the heavy reliance on serum creatinine in the AKI definition has several drawbacks. ${ }^{7}$ Although serum creatinine is routinely available and its measurement is standardized across institutions, creatinine may be affected by many nonrenal disease states, ${ }^{8-10}$ is a late marker of kidney function decline, and does not rise until a substantial amount of kidney function has been lost. ${ }^{11}$ As a result, the contributions of AKI to systemic diseases may be underappreciated because AKI is typically diagnosed late in the hospital course and may be incorrectly regarded as a consequence of systemic disease even though it may occur simultaneously or even before other complications. ${ }^{12}$ Third, oliguria is an excellent early marker of $\mathrm{AKI},{ }^{13}$ but it is less readily studied.

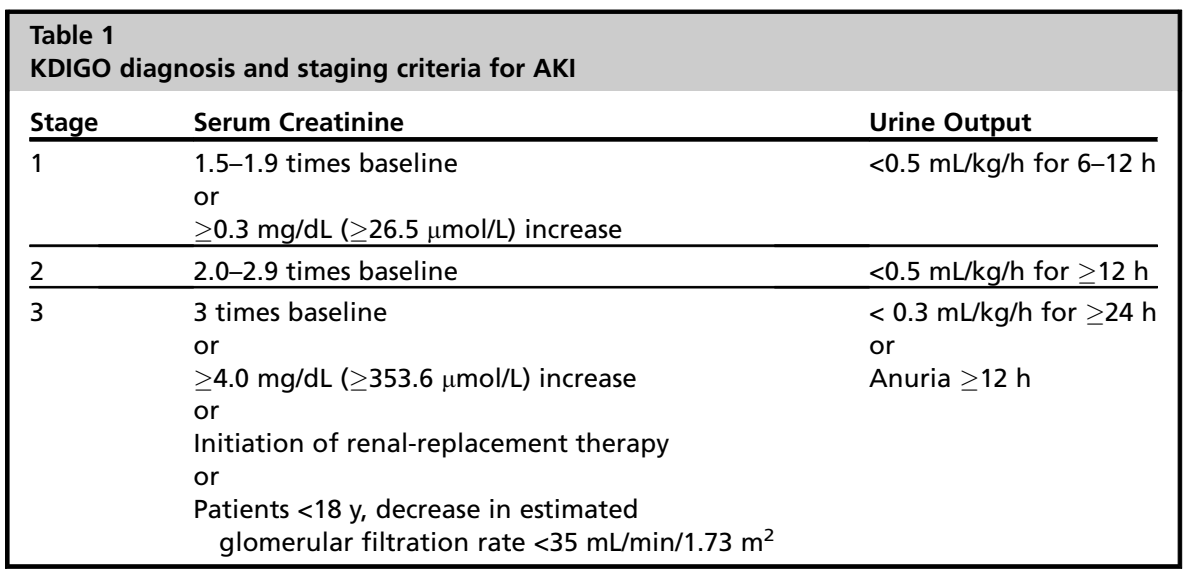

Data from Kellum JA, Lameire N, Group KAGW. Diagnosis, evaluation, and management of acute kidney injury: a KDIGO summary (Part 1). Crit Care. 2013;17(1):204. 


\section{CLINICAL OUTCOMES OF ACUTE KIDNEY INJURY AND ACUTE RESPIRATORY DISTRESS SYNDROME}

$\mathrm{AKI}$ is a common complication and associated with a high mortality in the hospital and ICU settings. AKI may complicate up to $20 \%$ of all hospital admissions. ${ }^{14}$ In the ICU, up to $57 \%$ of patients develop $\mathrm{AKI}$, and approximately $13 \%$ require renal-replacement therapy (RRT). ${ }^{15,16}$ More importantly, AKI is associated with a high mortality, ${ }^{14}$ and an international study evaluating more than 23 countries and 54 ICUs found that the hospital mortality ranged between $40 \%$ and $60 \% .{ }^{17}$ Another multinational cross-sectional study investigating $\mathrm{AKI}$ using the KDIGO criteria demonstrated that $\mathrm{AKI}$ is an independent predictor of in-hospital mortality across all stages of AKI with exponential increase in hazard ratios from mild/stage 1 disease (hazard ratio, 1.7) to severe/stage 3 disease (hazard ratio, 6.7) even after adjustment for covariates. ${ }^{15}$ Strong associations with mortality in $\mathrm{AKI}$ is also true across many different settings and populations, including aortic surgery, ${ }^{18}$ cardiac surgery, ${ }^{19}$ decompensated cirrhosis, ${ }^{20}$ and bone marrow transplant. ${ }^{21}$ Furthermore, AKI can increase the risk of long-term adverse outcomes with one large systematic review demonstrating an increased risk of mortality, myocardial infarction, and development of end-stage renal disease. ${ }^{22}$

\section{Clinical Outcomes of Acute Kidney Injury Complicating Acute Respiratory Distress Syndrome}

AKI is a common complication in patients with ARDS. A secondary analysis from the landmark ARDSnet trial demonstrated that approximately $24 \%$ of participants with ARDS developed AKI. ${ }^{23}$ One prospective, multicenter ICU study showed that $44.3 \%$ of ARDS patients also had AKI with a median time to diagnosis of 2 days after ARDS. ${ }^{24}$ After adjustment for cofounders, mechanical ventilation (MV) with ARDS had a high likelihood of developing AKI.

AKI complicating ARDS portends a poor prognosis. In the ARDSnet trial, the 180day mortality rate was much higher in those with AKI versus those without $(58 \%$ vs $28 \%)^{25}$ and this association was confirmed in other prospective studies. ${ }^{24}$ Similarly, another study evaluating oliguric renal failure and lung injury found that the survival rate was much lower compared with the entire cohort of patients studied (Fig. 1). ${ }^{26}$

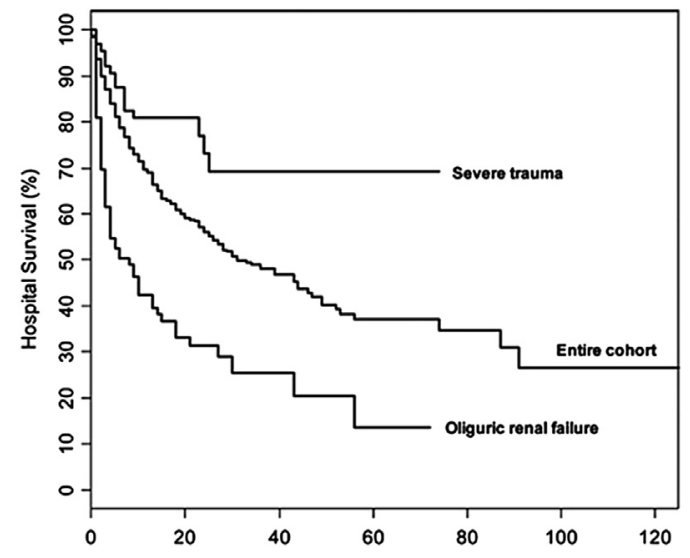

Fig. 1. ARDS complicated by severe AKI has increased mortality compared with ARDS alone. (Adapted from Cooke CR, Kahn JM, Caldwell E, et al. Predictors of hospital mortality in a population-based cohort of patients with acute lung injury. Crit Care Med. 2008;36(5):1412-1420; with permission.) 
Severe AKI requiring RRT was associated with up to $50 \%$ mortality, ${ }^{27}$ and one retrospective study demonstrated increased ventilator days (10 vs 7 days) and duration of weaning ( 41 vs 21 hours) in those with ARDS complicated by AKI versus ARDS alone. ${ }^{28}$

\section{THE EFFECT OF ACUTE KIDNEY INJURY ON THE LUNGS}

Traditional complications of AKI, such as electrolyte derangements, uremia, and fluid overload, have long been considered to contribute to the poor pulmonary outcomes associated with $\mathrm{AKI}$; however, research over the last two decades highlights the importance of nontraditional consequences of AKI (Table 2). ${ }^{29}$ The importance of nontraditional complications to outcomes after AKI is evidenced by the fact that RRT is well known to correct the traditional complications of AKI, yet the mortality of $\mathrm{AKI}$ requiring RRT in the ICU is $50 \%$ to $60 \% .^{30-33}$ Thus, improving mortality rates in patients with AKI requires therapies targeted beyond modifications and improvements to RRT.

$\mathrm{AKI}$ and its effects on the lungs has been well studied in animal models. ${ }^{34,35} \mathrm{AKI}-$ mediated lung injury is associated with lung inflammation characterized by increased levels of pulmonary cytokines, chemokines, and neutrophil accumulation. ${ }^{36-39}$ The proinflammatory cytokine interleukin (IL)- 6 increases in the plasma by 2 hours of $\mathrm{AKI}^{36,37}$ and is a major mediator of lung inflammation post-AKI. ${ }^{36,40}$ These findings are clinically relevant because patients with AKI develop increased plasma IL-6 within 2 hours ${ }^{41}$ and increased IL-6 is associated with prolonged MV ${ }^{41}$ and increased mortality. ${ }^{42}$ Additional characteristics of AKI-mediated lung injury in animal models include dysregulation of salt and water channels, ${ }^{43}$ pulmonary vascular congestion, ${ }^{39}$ T-cell accumulation, ${ }^{44}$ and apoptotic and necrotic cell death. ${ }^{45,46}$ Unlike direct lung injury, AKI lung injury is not characterized by significant epithelial injury and the alveolar space is devoid of inflammatory cytokines and neutrophils. ${ }^{47}$

\section{THE EFFECT OF ACUTE RESPIRATORY DISTRESS SYNDROME ON THE KIDNEYS}

Around the time of the ARDSnet trial, several papers demonstrated that protective lung strategies were associated with reduced serum cytokine/chemokine levels and

\begin{tabular}{|lc}
\hline \multicolumn{2}{|l}{ Table 2} \\
Traditional and nontraditional complications of AKI \\
\hline Traditional Complications of AKI & Nontraditional Complications of AKI \\
\hline Recognized for $>50 \mathrm{y}$ & Newly appreciated and studied in the past $20 \mathrm{y}$ \\
\hline $\begin{array}{l}\text { May contribute to increased } \\
\text { mortality of AKI }\end{array}$ & May contribute greatly to AKI mortality \\
\hline Typically corrected by renal-replacement & Requires therapy beyond renal-replacement \\
therapy & therapy \\
\hline Include & Include \\
Hyperkalemia & Respiratory complications/inflammatory lung \\
Acidosis & injury \\
Hyperphosphatemia & Sepsis \\
Hypocalcemia & Cardiac dysfunction/injury \\
Fluid overload & Intestinal injury \\
Pericarditis & Liver injury \\
Uremic bleeding & Immunoparalysis \\
\hline
\end{tabular}

Adapted from Faubel S, Edelstein CL. Mechanisms and mediators of lung injury after acute kidney injury. Nat Rev Nephrol. 2016;12(1):48-60; with permission. 
decreased organ dysfunction, including a reduced rate of $A K I .{ }^{23,48,49}$ The reduced rate of $\mathrm{AKI}$ with low tidal volume ventilation may be caused by the known effects of MV on renal function. ${ }^{50}$ Positive pressure ventilation was first shown to decrease renal perfusion in 1947. ${ }^{51}$ Since then, several studies in experimental models and clinical cohorts have shown that the use of positive end-expiratory pressure can decrease urine output likely caused by a reduction in cardiac output. ${ }^{52-55}$ Positive end-expiratory pressure has also been shown to alter the normal neurohormonal homeostasis (ie, renin-angiotensin-aldosterone axis) important for regulation of normal kidney function, ${ }^{55}$ resulting in decreased renal perfusion, glomerular filtration rate, and urine output. $^{52,55-57}$

\section{TREATMENT STRATEGIES FOR PATIENTS WITH ACUTE RESPIRATORY DISTRESS SYNDROME AND ACUTE KIDNEY INJURY}

Overall, treatment strategies for patients with ARDS and AKI are similar to the treatment of either condition alone. Next we discuss the general approach to ARDS and $\mathrm{AKI}$, and how care of one may influence overall treatment and physiology when the two are together.

\section{Acute Respiratory Distress Syndrome Management at a Glance}

In general, the identification and treatment of underlying causes for ARDS (eg, sepsis, trauma, and burns) will ensure optimal outcomes. The supportive treatment options for ARDS have been well-studied. ${ }^{58,59}$ First, the landmark ARDSnet trial ${ }^{23}$ showed a clinically significant reduction in mortality and more ventilator-free days with the use of low-tidal volume ventilation to prevent significant barotrauma. Second, among patients with severe ARDS, prone positioning significantly reduced 28-day mortality. ${ }^{60}$ Third, a conservative fluid management strategy with use of diuretics decreased ventilator-free days, reduced ICU days, and improved lung function, although a statistically significant mortality improvement was not appreciated. ${ }^{61,62}$

\section{Fluid Management in Acute Respiratory Distress Syndrome and Acute Kidney} Injury

Fluid overload has consistently been shown to be associated with adverse outcomes and worse mortality in the critically ill in general, and in patients with AKI in particular. ${ }^{63-65}$ Maintaining a net negative fluid balance (and therefore, less pulmonary edema) can positively affect lung physiology and outcomes in critically ill ventilated patients $^{61,66}$; however, clinical equipoise is key, and at some point striving for a net negative fluid balance is not beneficial once the patient's dry weight has been achieved. Several studies in septic shock and ARDS patients have demonstrated an association between a positive fluid balance and worse mortality, MV duration, and ICU length of stay. ${ }^{61,67-70}$ In the FACTT trial, the conservative fluid cohort (treated with diuretics) was more likely to have a shorter MV duration and shorter ICU stay compared with a liberal fluid strategy. ${ }^{61}$ There was also a trend for the conservative fluid group to require dialysis less often compared with the liberal fluid group highlighting that excess fluid administration does not protect against AKI requiring RRT as had been previously thought. Volume overload can also increase the risk of intra-abdominal hypertension and the risk of AKI through an overall reduction in renal blood flow. ${ }^{71,72}$ In the absence of intra-abdominal hypertension, excess volume can also increase edema in the renal interstitium thereby leading to worse AKI. ${ }^{68}$ 


\section{Acute Kidney Injury Management at a Glance}

The 2012 KDIGO recommendations for the management of AKI has been widely adopted and serve to help the clinician with prognostication and diagnostic/treatment decisions. ${ }^{6}$ Since its publication, several studies have shown that implementation of these guidelines may aid in prompt diagnosis and management of $\mathrm{AKI}$ in susceptible populations leading to improved clinical outcomes. ${ }^{73-7}$

The first step is to obtain an accurate diagnosis of AKI and identify the cause of kidney injury whenever possible. Next, the prevention of worsening injury revolves around maintaining adequate organ perfusion, avoiding volume overload, avoiding hyperglycemia, discontinuing nephrotoxic agents, and renally dosing medications. Lastly, when such maneuvers are inadequate and the patient develops worsening complications of $\mathrm{AKI}$ (eg, fluid overload, hyperkalemia), RRT is the next appropriate treatment modality to consider.

\section{Approach to Renal-Replacement Therapy in the Critically III Patient with Acute Kidney Injury}

Initiation of RRT requires astute clinical judgment and the collaboration of nephrologists and intensivists to determine patient suitability. In general, the decision to start RRT depends on (1) the underlying cause, (2) indications for RRT, (3) patient factors guiding modality, and (4) specific treatment variables. We briefly summarize important aspects of RRT for the critical care provider.

First, it is widely accepted that RRT should be initiated in those with severe electrolyte derangements (eg, hyperkalemia), severe acidosis, severe uremia, and pulmonary edema in the setting of oliguria. ${ }^{6}$ Even with this recommendation, clinical equipoise must be maintained and current guidelines also recommend considering the broader clinical context when starting dialysis.

Second, the timing of RRT has been well-studied and the current evidence indicates that early initiation has no benefits regarding survival. In general, most randomized controlled trials showed no mortality benefit with an early versus late timing approach to RRT. ${ }^{30,31,33,76,77}$ A meta-analysis of seven different randomized clinical trials with 1343 patients showed no benefit with an early RRT approach (95\% confidence interval, 0.74 [0.43-1.27]). ${ }^{78}$ As such, the ultimate timing decision depends on multiple factors to be addressed and discussed among nephrologists and intensivists.

Third, there is no firm consensus on the optimal RRT modality in AKI. Continuous RRT (CRRT) is generally preferred over intermittent hemodialysis and prolonged intermittent RRT because of perceived hemodynamic instability in the critically ill. One recent meta-analysis comparing CRRT, intermittent hemodialysis, and prolonged intermittent RRT showed no clear advantage of one modality over another on shortterm mortality and dialysis dependence. ${ }^{79}$

\section{Hypophosphatemia in Continuous Renal-Replacement Therapy}

CRRT is associated with a high incidence of severe hypophosphatemia occurring in up to $70 \%$ of patients. ${ }^{80-82}$ Phosphate is essential for all cells and is important for cell membrane integrity, bone structure, cell signaling, acid-base buffering, and energy storage in the form of adenosine triphosphate. ${ }^{83}$ As such, severe hypophosphatemia has been implicated in respiratory muscle failure and prolonged duration of MV. ${ }^{84-86}$ Additionally, reduced levels of phosphate can impair myocardial contractility and lead to arrhythmias, which may be improved once hypophosphatemia is corrected. ${ }^{83}$ Hypophosphatemia has been associated with prolonged $\mathrm{MV},{ }^{87}$ longer vasopressor duration, ${ }^{87}$ longer duration of CRRT use, ${ }^{82}$ longer ICU stays, ${ }^{82}$ and higher 
doses of CRRT. ${ }^{88}$ Therefore, it is important to monitor and treat the common complication of hypophosphatemia in RRT patients.

\section{ACUTE KIDNEY INJURY AND SARS-COV-2: CURRENT KNOWLEDGE}

SARS-CoV-2 is a novel coronavirus that was first reported in December $2019,{ }^{89}$ and has since become the most significant pandemic in the modern era. Initial reports suggested that the rates of $A K I$ were low ${ }^{90,91}$; however, more recent data suggest $A K I$ to be a common complication with values reported as high as 37\%. ${ }^{91-94}$ The ICU incidence of $\mathrm{AKI}$ is more significantly elevated at more than $50 \%$ in multiple studies, and has been associated with significant mortality. ${ }^{93,95,96}$ A large registry from the European Renal Association-European Dialysis and Transplant Association has shown a high short-term mortality rate of $20 \%$ for dialysis and renal transplant patients. ${ }^{97}$ Independent risk factors for AKI included elderly, Black race, diabetes, cardiovascular disease, hypertension, MV, and vasopressor use. ${ }^{93,98}$

The pathophysiologic mechanism underlying AKI in COVID-19 is still incompletely understood. The best evidence to date indicates that the underlying mechanism is similar to severe sepsis with one case series reporting acute tubular necrosis in approximately $66 \%$ of cases. ${ }^{96}$ Another important consideration is the cytokine storm phenomenon experienced in severe COVID-19 ARDS patients, which may lead to hypotension and sepsis further compromising renal perfusion. Focal kidney fibrin thrombi have been identified in histologic specimens, but are not currently thought to directly contribute to $\mathrm{AKI}$ and are instead considered a sequelae of deranged coagulopathy. ${ }^{99}$ AKI in COVID-19 patients may also be as a result of prerenal azotemia and tubular injury as a result of toxic insults, such as rhabdomyolysis. ${ }^{100}$ Collapsing glomerulopathy is an uncommon, but well-established cause of AKI that is associated with nephrotic syndrome and has been described particularly in the setting of high-risk APOL1 alleles. ${ }^{101}$ Other pathologic features described include membranous glomerulopathy, antiglomerular basement membrane nephritis, and exacerbation of preexisting autoimmune glomerulonephritis, but it is unclear whether these features are related to COVID-19 or new/preexisting diagnoses. ${ }^{102}$

Whether SARS-CoV-2 causes direct viral injury to the kidneys is currently controversial. Because SARS-CoV-2 enters cells via the ACE-2 receptors, which are abundant on the renal proximal tubule, it was thought that directly viral entry was probable. Early studies demonstrated viral staining in the proximal tubule, but later studies failed to confirm this. Targeting of ACE-2 receptors by COVID-19 may result in several downstream effects, such as hypercoagulation, innate and adaptive immune pathway activation, and angiotensin dysregulation. ${ }^{103}$ However, these studies also report on patient samples that did not demonstrate significant viral particle staining.

At time of submission, there are two therapies approved for use in severe COVID-19 illness (remdesivir and dexamethasone). ${ }^{104}$ Of note, remdesivir is currently contraindicated in those with a reduced glomerular filtration rate, but recent evidence suggests that it may be suitable in those receiving RRT. ${ }^{105}$ The initial concern for remdesivir use in patients with AKI revolved around the nephrotoxic accumulation of sulfobutylether$\beta$-cyclodextrin, but evidence suggests there is adequate removal of sulfobutylether$\beta$-cyclodextrin with dialysis. ${ }^{105,106}$ The risk of venous thromboembolism seems to be higher in this syndrome; however, recent critical care guidelines recommend against full anticoagulation without evidence of venous thromboembolisms, and recommend typical thromboprophylaxis and monitoring as key. ${ }^{107}$

The indications for RRT in the management of severe AKI in the setting of COVID-19 disease are the same as for other critically ill patients. ${ }^{94,108}$ One important distinction is 
the use of anticoagulation because higher incidence of filter clotting during CRRT in COVID-19 disease has been reported. ${ }^{109-111}$ Several studies have reported distinct perturbations in the clotting cascade in COVID-19 patients including thrombocytopenia and prolonged prothrombin/partial thromboplastic time, which may contribute to the high incidence of filter clotting. ${ }^{110,112,113}$ CRRT filter clotting is an important concern because it can lead to blood loss and lost time on RRT. ADQI guidelines for the management of AKI in COVID-19 patients recommend the use of anticoagulation if not otherwise contraindicated, monitoring for impending signs of circuit failure, and establishing center-specific stepwise escalation options for CRRT anticoagulation. ${ }^{94}$ Finally, as the pandemic continues, there is concern about dialysis and CRRT availability, including consumables, machines, and staff. ${ }^{114}$ Critical shortages were seen during the initial surge in New York City and similarly experienced abroad; therefore, preparation of resources in the coming months is key. ${ }^{114,115}$

\section{SUMMARY}

$\mathrm{AKI}$ is a common complication during hospital and ICU stays, and is particularly problematic when coexisting with ARDS. Previous studies have highlighted that $\mathrm{AKI}$ is an independent predictor for death in patients who are critically ill with acute lung injury. Clinical and experimental data indicate that there is significant crosstalk between injured kidneys and the lung, and that AKI exerts a multitude of deleterious effects on the lung via fluid overload leading to cardiogenic pulmonary edema, cytokine excess leading to noncardiogenic pulmonary edema, and others. The organ-organ effects of kidney and lung injury have been especially poignant in the era of the novel SARS-CoV-2 virus where the existence of both complications portends a poor prognosis. In summary, AKI complicating ARDS is a common phenomenon that contributes to a significant burden of disease, and clinical recognition of this syndrome aids the clinician in management and prognostication in the critically ill.

\section{CRITICAL CARE POINTS}

- AKI in conjunction with ARDS portends a poor prognosis and can help guide the intensivist in goals of care discussion.

- AKI can affect the lungs in multiple ways via traditional (eg, volume overload) and nontraditional (eg, systemic inflammatory mediators) complications.

- Maintaining appropriate fluid balance and ensuring adequate treatment of the underlying cause of ARDS are crucial.

- If a patient becomes anuric, renal-replacement therapy should be considered. Initiation and management of such treatment should involve a continuous dialogue between the intensivist and nephrologist.

- AKI is also a common complication of COVID-19. In general, management of AKI in COVID-19 patients is similar to other disease states. However, special consideration should be made to potential drug toxicities of new SARS-CoV2 agents; and the increased prevalence of hypercoagulability in this population.

\section{DISCLOSURE}

None. 


\section{REFERENCES}

1. Liu KD, Matthay MA. Advances in critical care for the nephrologist: acute lung injury/ARDS. Clin J Am Soc Nephrol 2008;3(2):578-86.

2. Liu KD, Thompson BT, Ancukiewicz M, et al. Acute kidney injury in patients with acute lung injury: impact of fluid accumulation on classification of acute kidney injury and associated outcomes. Crit Care Med 2011;39(12):2665-71.

3. Mehta RL, Pascual MT, Gruta CG, et al. Refining predictive models in critically ill patients with acute renal failure. J Am Soc Nephrol 2002;13(5):1350-7.

4. Mehta RL, Kellum JA, Shah SV, et al. Acute Kidney Injury Network: report of an initiative to improve outcomes in acute kidney injury. Crit Care 2007;11(2):R31.

5. Bellomo R, Ronco C, Kellum JA, et al. Acute Dialysis Quality Initiative w. Acute renal failure - definition, outcome measures, animal models, fluid therapy and information technology needs: the Second International Consensus Conference of the Acute Dialysis Quality Initiative (ADQI) Group. Crit Care 2004;8(4): R204-12.

6. Kellum JA, Lameire N, Group KAGW. Diagnosis, evaluation, and management of acute kidney injury: a KDIGO summary (Part 1). Crit Care 2013;17(1):204.

7. Selby NM, Fluck RJ, Kolhe NV, et al. International criteria for acute kidney injury: advantages and remaining challenges. PloS Med 2016;13(9):e1002122.

8. Caregaro L, Menon F, Angeli P, et al. Limitations of serum creatinine level and creatinine clearance as filtration markers in cirrhosis. Arch Intern Med 1994; 154(2):201-5.

9. Racz O, Lepej J, Fodor B, et al. Pitfalls in the measurements and assessment of glomerular filtration rate and how to escape them. EJIFCC 2012;23(2):33-40.

10. Lin J, Fernandez $H$, Shashaty MG, et al. False-positive rate of $A K I$ using consensus creatinine-based criteria. Clin J Am Soc Nephrol 2015;10(10): 1723-31.

11. Vaidya VS, Ferguson MA, Bonventre JV. Biomarkers of acute kidney injury. Annu Rev Pharmacol Toxicol 2008;48:463-93.

12. Palevsky PM, Liu KD, Brophy PD, et al. KDOQI US commentary on the 2012 KDIGO clinical practice guideline for acute kidney injury. Am J Kidney Dis 2013;61(5):649-72.

13. Macedo E, Malhotra R, Bouchard J, et al. Oliguria is an early predictor of higher mortality in critically ill patients. Kidney Int 2011;80(7):760-7.

14. Uchino S, Bellomo R, Goldsmith D, et al. An assessment of the RIFLE criteria for acute renal failure in hospitalized patients. Crit Care Med 2006;34(7):1913-7.

15. Hoste EA, Bagshaw SM, Bellomo R, et al. Epidemiology of acute kidney injury in critically ill patients: the multinational AKI-EPI study. Intensive Care Med 2015; 41(8): 1411-23.

16. Nisula S, Kaukonen KM, Vaara ST, et al. Incidence, risk factors and 90-day mortality of patients with acute kidney injury in Finnish intensive care units: the FINNAKI study. Intensive Care Med 2013;39(3):420-8.

17. Uchino S, Kellum JA, Bellomo R, et al. Acute renal failure in critically ill patients: a multinational, multicenter study. J Am Med Assoc 2005;294(7):813-8.

18. Kashyap VS, Cambria RP, Davison JK, et al. Renal failure after thoracoabdominal aortic surgery. J Vasc Surg 1997;26(6):949-55, discussion 955-947.

19. Chertow GM, Levy EM, Hammermeister KE, et al. Independent association between acute renal failure and mortality following cardiac surgery. Am J Med 1998;104(4):343-8. 
20. Aggarwal A, Ong JP, Younossi ZM, et al. Predictors of mortality and resource utilization in cirrhotic patients admitted to the medical ICU. Chest 2001;119(5): 1489-97.

21. Parikh CR, McSweeney P, Schrier RW. Acute renal failure independently predicts mortality after myeloablative allogeneic hematopoietic cell transplant. Kidney Int 2005;67(5):1999-2005.

22. Coca SG, Yusuf B, Shlipak MG, et al. Long-term risk of mortality and other adverse outcomes after acute kidney injury: a systematic review and meta-analysis. Am J Kidney Dis 2009;53(6):961-73.

23. Acute Respiratory Distress Syndrome N, Brower RG, Matthay MA, et al. Ventilation with lower tidal volumes as compared with traditional tidal volumes for acute lung injury and the acute respiratory distress syndrome. N Engl J Med 2000; 342(18): 1301-8.

24. Darmon M, Clec'h C, Adrie C, et al. Acute respiratory distress syndrome and risk of AKI among critically ill patients. Clin J Am Soc Nephrol 2014;9(8):1347-53.

25. Liu KD, Glidden DV, Eisner MD, et al. Predictive and pathogenetic value of plasma biomarkers for acute kidney injury in patients with acute lung injury. Crit Care Med 2007;35(12):2755-61.

26. Cooke CR, Kahn JM, Caldwell E, et al. Predictors of hospital mortality in a population-based cohort of patients with acute lung injury. Crit Care Med 2008;36(5):1412-20.

27. Dill J, Bixby B, Ateeli H, et al. Renal replacement therapy in patients with acute respiratory distress syndrome: a single-center retrospective study. Int J Nephrol Renovasc Dis 2018;11:249-57.

28. Vieira JM Jr, Castro I, Curvello-Neto A, et al. Effect of acute kidney injury on weaning from mechanical ventilation in critically ill patients. Crit Care Med 2007;35(1):184-91.

29. Faubel S, Edelstein CL. Mechanisms and mediators of lung injury after acute kidney injury. Nat Rev Nephrol 2016;12(1):48-60.

30. Barbar SD, Clere-Jehl R, Bourredjem A, et al. Timing of renal-replacement therapy in patients with acute kidney injury and sepsis. N Engl J Med 2018;379(15): 1431-42.

31. Gaudry S, Hajage D, Schortgen F, et al. Initiation strategies for renalreplacement therapy in the intensive care Unit. N Engl J Med 2016;375(2): 122-33.

32. Metnitz PG, Krenn CG, Steltzer $H$, et al. Effect of acute renal failure requiring renal replacement therapy on outcome in critically ill patients. Crit Care Med 2002;30(9):2051-8.

33. Zarbock A, Kellum JA, Schmidt C, et al. Effect of early vs delayed initiation of renal replacement therapy on mortality in critically ill patients with acute kidney injury: the ELAIN randomized clinical trial. J Am Med Assoc 2016;315(20): 2190-9.

34. Grams ME, Rabb H. The distant organ effects of acute kidney injury. Kidney Int 2012;81(10):942-8.

35. Lee SA, Cozzi M, Bush EL, et al. Distant organ dysfunction in acute kidney injury: a review. Am J Kidney Dis 2018;72(6):846-56.

36. Klein CL, Hoke TS, Fang WF, et al. Interleukin-6 mediates lung injury following ischemic acute kidney injury or bilateral nephrectomy. Kidney Int 2008;74(7): 901-9. 
37. Hoke TS, Douglas IS, Klein CL, et al. Acute renal failure after bilateral nephrectomy is associated with cytokine-mediated pulmonary injury. J Am Soc Nephrol 2007; 18(1):155-64.

38. Awad AS, Rouse M, Huang L, et al. Compartmentalization of neutrophils in the kidney and lung following acute ischemic kidney injury. Kidney Int 2009;75(7): 689-98.

39. Kramer AA, Postler G, Salhab KF, et al. Renal ischemia/reperfusion leads to macrophage-mediated increase in pulmonary vascular permeability. Kidney Int 1999;55(6):2362-7.

40. Altmann C, Ahuja N, Kiekhaefer CM, et al. Early peritoneal dialysis reduces lung inflammation in mice with ischemic acute kidney injury. Kidney Int 2017;92(2): 365-76.

41. Liu KD, Altmann C, Smits G, et al. Serum interleukin-6 and interleukin-8 are early biomarkers of acute kidney injury and predict prolonged mechanical ventilation in children undergoing cardiac surgery: a case-control study. Crit Care 2009; 13(4):R104.

42. Simmons EM, Himmelfarb J, Sezer MT, et al. Plasma cytokine levels predict mortality in patients with acute renal failure. Kidney Int 2004;65(4):1357-65.

43. Rabb H, Wang Z, Nemoto $T$, et al. Acute renal failure leads to dysregulation of lung salt and water channels. Kidney Int 2003;63(2):600-6.

44. Lie ML, White LE, Santora RJ, et al. Lung T Iymphocyte trafficking and activation during ischemic acute kidney injury. J Immunol 2012;189(6):2843-51.

45. Nakazawa D, Kumar SV, Marschner J, et al. Histones and neutrophil extracellular traps enhance tubular necrosis and remote organ injury in ischemic AKI. J Am Soc Nephrol 2017;28(6):1753-68.

46. Hassoun HT, Lie ML, Grigoryev DN, et al. Kidney ischemia-reperfusion injury induces caspase-dependent pulmonary apoptosis. Am J Physiol Renal Physiol 2009;297(1):F125-37.

47. Bhargava R, Janssen W, Altmann C, et al. Intratracheal IL-6 protects against lung inflammation in direct, but not indirect, causes of acute lung injury in mice. PLoS ONE 2013;8(5):e61405.

48. Ranieri VM, Giunta F, Suter PM, et al. Mechanical ventilation as a mediator of multisystem organ failure in acute respiratory distress syndrome. J Am Med Assoc 2000;284(1):43-4.

49. Ranieri VM, Suter PM, Tortorella C, et al. Effect of mechanical ventilation on inflammatory mediators in patients with acute respiratory distress syndrome: a randomized controlled trial. J Am Med Assoc 1999;282(1):54-61.

50. Kuiper JW, Groeneveld AB, Slutsky AS, et al. Mechanical ventilation and acute renal failure. Crit Care Med 2005;33(6):1408-15.

51. Drury DR, Henry JP, Goodman J. The effects of continuous pressure breathing on kidney function. J Clin Invest 1947;26(5):945-51.

52. Annat G, Viale JP, Bui Xuan B, et al. Effect of PEEP ventilation on renal function, plasma renin, aldosterone, neurophysins and urinary $\mathrm{ADH}$, and prostaglandins. Anesthesiology 1983;58(2):136-41.

53. Pannu N, Mehta RL. Effect of mechanical ventilation on the kidney. Best Pract Res Clin Anaesthesiol 2004;18(1):189-203.

54. Priebe HJ, Heimann JC, Hedley-Whyte J. Mechanisms of renal dysfunction during positive end-expiratory pressure ventilation. J Appl Physiol Respir Environ Exerc Physiol 1981;50(3):643-9.

55. Koyner JL, Murray PT. Mechanical ventilation and the kidney. Blood Purif 2010; 29(1):52-68. 
56. Andrivet $P$, Adnot S, Sanker S, et al. Hormonal interactions and renal function during mechanical ventilation and ANF infusion in humans. J Appl Physiol (1985) 1991;70(1):287-92.

57. Bark H, Le Roith D, Nyska M, et al. Elevations in plasma ADH levels during PEEP ventilation in the dog: mechanisms involved. Am J Physiol 1980;239(6): E474-81.

58. Fan E, Del Sorbo L, Goligher EC, et al. An official American Thoracic Society/European Society of Intensive Care Medicine/Society of Critical Care Medicine clinical practice guideline: mechanical ventilation in adult patients with acute respiratory distress syndrome. Am J Respir Crit Care Med 2017;195(9): 1253-63.

59. Thompson BT, Chambers RC, Liu KD. Acute respiratory distress syndrome. N Engl J Med 2017;377(6):562-72.

60. Guerin C, Reignier J, Richard JC, et al. Prone positioning in severe acute respiratory distress syndrome. N Engl J Med 2013;368(23):2159-68.

61. National Heart L, Blood Institute Acute Respiratory Distress Syndrome Clinical Trials N, Wiedemann HP, et al. Comparison of two fluid-management strategies in acute lung injury. N Engl J Med 2006;354(24):2564-75.

62. Silversides JA, Major E, Ferguson AJ, et al. Conservative fluid management or deresuscitation for patients with sepsis or acute respiratory distress syndrome following the resuscitation phase of critical illness: a systematic review and meta-analysis. Intensive Care Med 2017;43(2):155-70.

63. Bagshaw SM, Brophy PD, Cruz D, et al. Fluid balance as a biomarker: impact of fluid overload on outcome in critically ill patients with acute kidney injury. Crit Care 2008;12(4):169.

64. Claure-Del Granado R, Mehta RL. Fluid overload in the ICU: evaluation and management. BMC Nephrol 2016;17(1):109.

65. Selewski DT, Goldstein SL. The role of fluid overload in the prediction of outcome in acute kidney injury. Pediatr Nephrol 2018;33(1):13-24.

66. Seeley EJ. A dry lung is a happy lung: more supporting evidence. J Thorac Cardiovasc Surg 2015;149(1):321-2.

67. Pittard MG, Huang SJ, McLean AS, et al. Association of positive fluid balance and mortality in sepsis and septic shock in an Australian cohort. Anaesth Intensive Care 2017;45(6):737-43.

68. Prowle JR, Echeverri JE, Ligabo EV, et al. Fluid balance and acute kidney injury. Nat Rev Nephrol 2010;6(2):107-15.

69. Rosenberg AL, Dechert RE, Park PK, et al. Review of a large clinical series: association of cumulative fluid balance on outcome in acute lung injury: a retrospective review of the ARDSnet tidal volume study cohort. J Intensive Care Med 2009;24(1):35-46.

70. van Mourik N, Metske HA, Hofstra JJ, et al. Cumulative fluid balance predicts mortality and increases time on mechanical ventilation in ARDS patients: an observational cohort study. PLoS One 2019;14(10):e0224563.

71. Dalfino L, Tullo L, Donadio I, et al. Intra-abdominal hypertension and acute renal failure in critically ill patients. Intensive Care Med 2008;34(4):707-13.

72. Vidal MG, Ruiz Weisser J, Gonzalez F, et al. Incidence and clinical effects of intra-abdominal hypertension in critically ill patients. Crit Care Med 2008; 36(6): 1823-31.

73. Meersch M, Schmidt C, Hoffmeier A, et al. Prevention of cardiac surgeryassociated $\mathrm{AKI}$ by implementing the KDIGO guidelines in high risk patients 
identified by biomarkers: the PrevAKI randomized controlled trial. Intensive Care Med 2017;43(11):1551-61.

74. Acosta-Ochoa I, Bustamante-Munguira J, Mendiluce-Herrero A, et al. Impact on outcomes across KDIGO-2012 AKI criteria according to baseline renal function. J Clin Med 2019;8(9):1323-36.

75. Gocze I, Jauch D, Gotz M, et al. Biomarker-guided intervention to prevent acute kidney injury after major surgery: the prospective randomized BigpAK study. Ann Surg 2018;267(6):1013-20.

76. Investigators S-A. Canadian Critical Care Trials G, Australian, et al. Timing of initiation of renal-replacement therapy in acute kidney injury. N Engl J Med 2020;383(3):240-51.

77. Wald R, Adhikari NK, Smith OM, et al. Comparison of standard and accelerated initiation of renal replacement therapy in acute kidney injury. Kidney Int 2015; 88(4):897-904.

78. Bagshaw SM, Wald R. Strategies for the optimal timing to start renal replacement therapy in critically ill patients with acute kidney injury. Kidney Int 2017; 91(5):1022-32.

79. Nash DM, Przech S, Wald R, et al. Systematic review and meta-analysis of renal replacement therapy modalities for acute kidney injury in the intensive care unit. J Crit Care 2017;41:138-44.

80. Yang $Y$, Zhang P, Cui Y, et al. Hypophosphatemia during continuous venovenous hemofiltration is associated with mortality in critically ill patients with acute kidney injury. Crit Care 2013;17(5):R205.

81. Song YH, Seo EH, Yoo YS, et al. Phosphate supplementation for hypophosphatemia during continuous renal replacement therapy in adults. Ren Fail 2019; 41(1):72-9.

82. Hendrix RJ, Hastings MC, Samarin M, et al. Predictors of hypophosphatemia and outcomes during continuous renal replacement therapy. Blood Purif 2020; 49(6):700-7.

83. Geerse DA, Bindels AJ, Kuiper MA, et al. Treatment of hypophosphatemia in the intensive care unit: a review. Crit Care 2010;14(4):R147.

84. Agusti AG, Torres A, Estopa R, et al. Hypophosphatemia as a cause of failed weaning: the importance of metabolic factors. Crit Care Med 1984;12(2):142-3.

85. Aubier M, Murciano D, Lecocguic Y, et al. Effect of hypophosphatemia on diaphragmatic contractility in patients with acute respiratory failure. N Engl J Med 1985;313(7):420-4.

86. Gravelyn TR, Brophy N, Siegert C, et al. Hypophosphatemia-associated respiratory muscle weakness in a general inpatient population. Am J Med 1988; 84(5):870-6.

87. Lim C, Tan HK, Kaushik M. Hypophosphatemia in critically ill patients with acute kidney injury treated with hemodialysis is associated with adverse events. Clin Kidney J 2017;10(3):341-7.

88. Bellomo R, Cass A, Cole L, et al. The relationship between hypophosphataemia and outcomes during low-intensity and high-intensity continuous renal replacement therapy. Crit Care Resusc 2014;16(1):34-41.

89. Zhu N, Zhang D, Wang W, et al. A novel coronavirus from patients with pneumonia in China, 2019. N Engl J Med 2020;382(8):727-33.

90. Wang L, Li X, Chen H, et al. Coronavirus disease 19 infection does not result in acute kidney injury: an analysis of 116 hospitalized patients from Wuhan, China. Am J Nephrol 2020;51(5):343-8. 
91. Guan WJ, Ni ZY, Hu Y, et al. Clinical characteristics of coronavirus disease 2019 in China. N Engl J Med 2020;382(18):1708-20.

92. Chen N, Zhou M, Dong X, et al. Epidemiological and clinical characteristics of 99 cases of 2019 novel coronavirus pneumonia in Wuhan, China: a descriptive study. Lancet 2020;395(10223):507-13.

93. Hirsch JS, Ng JH, Ross DW, et al. Acute kidney injury in patients hospitalized with COVID-19. Kidney Int 2020;98(1):209-18.

94. Nadim MK, Forni LG, Mehta RL, et al. COVID-19-associated acute kidney injury: consensus report of the 25th Acute Disease Quality Initiative (ADQI) Workgroup. Nat Rev Nephrol 2020;16(12):747-64.

95. Chan L, Chaudhary K, Saha A, et al. Acute kidney injury in hospitalized patients with COVID-19. medRxiv 2020.

96. Mohamed MMB, Lukitsch I, Torres-Ortiz AE, et al. Acute kidney injury associated with coronavirus disease 2019 in urban New Orleans. Kidney360. 2020; 1(7):614-22.

97. Jager KJ, Kramer A, Chesnaye NC, et al. Results from the ERA-EDTA Registry indicate a high mortality due to COVID-19 in dialysis patients and kidney transplant recipients across Europe. Kidney Int 2020;98(6):1540-8.

98. Xia $P$, Wen $Y$, Duan $Y$, et al. Clinicopathological features and outcomes of acute kidney injury in critically ill COVID-19 with prolonged disease course: a retrospective cohort. J Am Soc Nephrol 2020;31(9):2205-21.

99. Santoriello D, Khairallah P, Bomback AS, et al. Postmortem kidney pathology findings in patients with COVID-19. J Am Soc Nephrol 2020;31(9):2158-67.

100. Sharma P, Uppal NN, Wanchoo R, et al. COVID-19-associated kidney injury: a case series of kidney biopsy findings. J Am Soc Nephrol 2020;31(9):1948-58.

101. Wu H, Larsen CP, Hernandez-Arroyo CF, et al. AKI and collapsing glomerulopathy associated with COVID-19 and APOL 1 high-risk genotype. J Am Soc Nephrol 2020;31(8):1688-95.

102. Kudose S, Batal I, Santoriello D, et al. Kidney biopsy findings in patients with COVID-19. J Am Soc Nephrol 2020;31(9):1959-68.

103. Batlle D, Soler MJ, Sparks MA, et al. Acute kidney injury in COVID-19: emerging evidence of a distinct pathophysiology. J Am Soc Nephrol 2020;31(7):1380-3.

104. Panel C-TG. Coronavirus disease 2019 (COVID-19) treatment guidelines. National Institutes of Health. Available at: https://www.covid19treatment guidelines.nih.gov/. Accessed: December 5, 2020.

105. Thakare S, Gandhi C, Modi T, et al. Safety of remdesivir in patients with acute kidney injury or CKD. Kidney Int Rep 2021;6(1):206-10.

106. Kiser TH, Fish DN, Aquilante CL, et al. Evaluation of sulfobutylether-betacyclodextrin (SBECD) accumulation and voriconazole pharmacokinetics in critically ill patients undergoing continuous renal replacement therapy. Crit Care 2015;19:32.

107. Moores LK, Tritschler T, Brosnahan S, et al. Prevention, diagnosis, and treatment of VTE in patients with coronavirus disease 2019: CHEST guideline and Expert Panel report. Chest 2020;158(3):1143-63.

108. Panel C-TG. Coronavirus disease 2019 (COVID-19) treatment guidelines. National Institutes of Health. Available at: https://www.covid19treatment guidelines.nih.gov/. Accessed August 23, 2020.

109. Helms J, Tacquard C, Severac F, et al. High risk of thrombosis in patients with severe SARS-CoV-2 infection: a multicenter prospective cohort study. Intensive Care Med 2020;46(6):1089-98. 
110. Endres P, Rosovsky R, Zhao S, et al. Filter clotting with continuous renal replacement therapy in COVID-19. J Thromb Thrombolysis 2021;51(4):966-70.

111. Shankaranarayanan D, Muthukumar T, Barbar T, et al. Anticoagulation strategies and filter life in COVID-19 patients receiving continuous renal replacement therapy: a single-center experience. Clin J Am Soc Nephrol 2020;16(1):124-6.

112. Cui S, Chen S, Li X, et al. Prevalence of venous thromboembolism in patients with severe novel coronavirus pneumonia. J Thromb Haemost 2020;18(6): $1421-4$.

113. Klok FA, Kruip M, van der Meer NJM, et al. Incidence of thrombotic complications in critically ill ICU patients with COVID-19. Thromb Res 2020;191:145-7.

114. Hsu CM, Weiner DE. COVID-19 in dialysis patients: outlasting and outsmarting a pandemic. Kidney Int 2020;98(6):1402-4.

115. Mogul F. Shortage of dialysis equipment leads to difficult decisions in New York ICUs [press release]. Natl Public Radio 2020;2020. 